\title{
Robotics and the spine: a review of current and ongoing applications
}

\author{
Faris Shweikeh, B.S., ${ }^{1}$ Jordan P. Amadio, M.D., M.B.A., ${ }^{3}$ Monica Arnell, B.A., ${ }^{1}$ \\ Zachary R. Barnard, M.D., ${ }^{1}$ Terrence T. Kim, M.D., ${ }^{2}$ J. Patrick Johnson, M.D., ${ }^{1,4}$ \\ and Doniel Drazin, M.D. ${ }^{1}$
}

Departments of ${ }^{1}$ Neurosurgery and ${ }^{2}$ Orthopedics, Cedars-Sinai Medical Center, Los Angeles; ${ }^{4}$ Department of Neurosurgery, University of California Davis Medical Center, Sacramento, California; and ${ }^{3}$ Department of Neurosurgery, Emory University, Atlanta, Georgia

\begin{abstract}
Object. Robotics in the operating room has shown great use and versatility in multiple surgical fields. Robotassisted spine surgery has gained significant favor over its relatively short existence, due to its intuitive promise of higher surgical accuracy and better outcomes with fewer complications. Here, the authors analyze the existing literature on this growing technology in the era of minimally invasive spine surgery.

Methods. In an attempt to provide the most recent, up-to-date review of the current literature on robotic spine surgery, a search of the existing literature was conducted to obtain all relevant studies on robotics as it relates to its application in spine surgery and other interventions.

Results. In all, 45 articles were included in the analysis. The authors discuss the current status of this technology and its potential in multiple arenas of spinal interventions, mainly spine surgery and spine biomechanics testing.

Conclusions. There are numerous potential advantages and limitations to robotic spine surgery, as suggested in published case reports and in retrospective and prospective studies. Randomized controlled trials are few in number and show conflicting results regarding accuracy. The present limitations may be surmountable with future technological improvements, greater surgeon experience, reduced cost, improved operating room dynamics, and more training of surgical team members. Given the promise of robotics for improvements in spine surgery and spine biomechanics testing, more studies are needed to further explore the applicability of this technology in the spinal operating room. Due to the significant cost of the robotic equipment, studies are needed to substantiate that the increased equipment costs will result in significant benefits that will justify the expense.
\end{abstract}

(http://thejns.org/doi/abs/10.3171/2014.1.FOCUS13526)

KEY WoRdS • robot • robotics • spine surgery • biomechanics

$\mathrm{T}$ Echnological advances have revolutionized spine surgery over the past 20 years. Many of these developments have been centered on the innovations of new technology implants and minimally invasive spine surgery. The goals of these improvements have been to balance the maintenance of a high degree of precision, to minimize risks of damage to neurovascular structures, to facilitate surgeon access and operating room dynamics, and to diminish harmful exposure to ionizing radiation in patients and the operative team. Because the risks associated with spine surgery are plentiful and limiting complications is imperative, implementing a robot-assisted technique has the potential to address many concerns associated with conventional surgery.

Robotic systems have been used in multiple surgical disciplines including gynecology, urology, and general surgery. In the last decade we have seen the application of this technology to spine surgery. Robots can potentially

Abbreviations used in this paper: $\mathrm{RCT}=$ randomized controlled trial; $\mathrm{ROM}=$ range of motion. help with all matters of spine surgery including the following: precision in spinal instrumentation, eliminating dural and neurovascular injuries, minimizing exposure to radiation, and improving operating room workflow. ${ }^{5}$ At the same time, the technology affords the surgeon a significant improvement in coordination, 3D visualization, and a reduction in fatigue, and it offers the patient a smaller incision, lower risk of infections, minimal muscle retraction and thus postoperative pain, and shortened length of hospital stay. ${ }^{16}$ Apart from screw and rod insertion, the technology has been applied to other forms of spine operations and pathological conditions including tumor resections and ablations, vertebroplasties, and anesthetic blocks. ${ }^{5}$

This article provides an overview and update on the utility of robotics in spine surgery and spine biomechanics testing.

\section{Methods}

Using the National Library of Medicine search en- 
gine, PubMed, we searched the literature to identify articles that had been published between 1950 and 2013 that were pertinent to robotics in spine surgery. The key words used in the search were "spine" and/or "spinal" and/or "surgery" and/or "robotics" and/or "robots." Relevant primary articles describing case reports or clinical studies were selected, and the reference lists from these articles were also inspected for other relevant articles. Each of the resultant articles was reviewed by all authors, discussed in a committee, and reported in this review. Reviews and editorials were excluded. Only publications in Englishlanguage, peer-reviewed journals were included. Articles were then categorized into cadaveric, human clinical, and biomechanics studies.

\section{Results}

In all, 45 articles were included. The following is a review and analysis of the 45 published reports and studies regarding robotics in spine surgery and spine biomechanics testing.

\section{Robotics and the Operating Theater: A Match Made in Heaven}

Since the advent of modern surgical robots in the early 1990s, robotic technology has become well established in urology, gynecology, and general surgery for its advantages in laparoscopic procedures. ${ }^{6,28,44}$ The da Vinci surgical system (Intuitive Surgical, Inc.) was approved by the FDA in 2000 for use in general surgery operations, in 2001 for urological operations, and in 2005 for gynecological surgery. ${ }^{1,22,44}$ In 2010 alone, more than 300,000 robotic procedures were completed, and exploration continues to find further avenues for use. ${ }^{44}$ Over the past decade, several advances have been made in robotic technology following years of experience with these robots in the operating room. ${ }^{1,44}$ One of the most widespread uses of robotic technology has developed in urology. Although both robot-assisted and laparoscopic prostatectomy have been shown to reduce blood loss and hospital stay significantly when compared with open prostatectomy, robotic technology has demonstrated a reduced learning curve and improved intraoperative visualization when compared with laparoscopy. ${ }^{4}$ Additional examples include high-definition visualization for small operative areas, robot accessibility in the operating room, and haptic feedback (the ability to inform surgeons regarding pressure and touch when using instruments). ${ }^{44}$

Over the past decade, robotic technology has been increasingly used in spine surgery, with a rapidly expanding breadth of applications. ${ }^{2,9,39}$ Several models are under research and development; for instance, the da Vinci surgical system has been used for paraspinal tumor removal and transoral odontoidectomy under a research protocol, with great success. ${ }^{5,23,24,30,34,45}$ SpineAssist (Mazor Surgical Technologies) is a miniature robot approved by the FDA for pedicle screw placement, which has been evaluated in multiple retrospective and a few prospective studies. ${ }^{7,915}$ Such robots have been incorporated in the spinal operating theater for the theoretical benefits of improved visualization, reduced radiation exposure for both pa- tients and surgeons, and improved accuracy in placement of screws and implants. ${ }^{915}$ However, because this technology is increasingly used in the field, it will be important to provide evidence that the use of robot-assisted surgery is superior to freehand or image-guided methods in patient outcomes and cost-effectiveness. ${ }^{35-37}$

Many factors specific to surgery in the spine make the use of robotic technology ideal for improvement of techniques and outcomes. Placement of screws and implants can be assisted by robotic localization for accuracy and precision. ${ }^{36,39}$ The proximity of blood vessels and nerve roots to the bony structure creates the possibility of severe postoperative complications should errors occur. ${ }^{39}$ Procedures in the spine thus require fine motor coordination, which may be assisted by robotic minimization of hand tremor. ${ }^{36}$ Access to the spine and visualization through a long corridor are often difficult. ${ }^{33}$ Minimally invasive surgery has been shown to improve outcomes and recovery rates in addition to reducing complications, infection, blood loss, and tissue trauma. ${ }^{36}$ However, these procedures often require much longer periods of exposure to radiation for both surgeons and patients, causing many surgeons to rely on a standard open approach rather than a minimally invasive approach to the spine. From 2004 to 2007, a retrospective review of more than 100,000 spine cases showed that only $13.2 \%$ were minimally invasive in approach. ${ }^{11}$ Should robotic spine surgery provide an avenue for minimally invasive surgery while reducing intraoperative exposure to radiation and improving accuracy of screw placement, it may be considered a future standard for improving outcomes for both patients and surgeons. ${ }^{33,36,39}$

\section{Cadaveric Studies of Robot-Assisted Spine Surgery}

Paramount to assessing assumptions about robotic applications in spine surgery, as with any new surgical innovation, is how to test feasibility and efficacy. Although the hypothesized advantages and disadvantages robot-assisted spine surgery are plausible, they have not been delineated in a systematic approach. Efficacy is classically determined by quality designed clinical trials. However, there are many hurdles to designing clinical trials that involve surgical intervention-including randomization, blinding, and ethical concerns. Prior to testing new surgical techniques or equipment, feasibility studies must be completed to evaluate whether the aim of the new intervention is possible. The most convincing studies on the feasibility of robot-assisted spine surgery have been performed on human cadavers. The use of cadavers for this purpose has advantages and disadvantages. Although cadaveric anatomy is the same, there is no way to identify complications except by radiographic imaging after placement of instrumentation. Other outcomes and complications including pain, relief of symptoms, quality of life, bleeding, nerve root injuries, and more cannot be assessed. Table 1 illustrates the variety of cadaveric studies that have been performed and their characteristics. ${ }^{18,19,21,25-27,38,40}$

Whereas many published studies consisted of $1-2$ cadavers, Togawa et al. ${ }^{42}$ reported on the placement of lumbosacral pedicle screws, translaminar facet screws, and 
Robotics and the spine

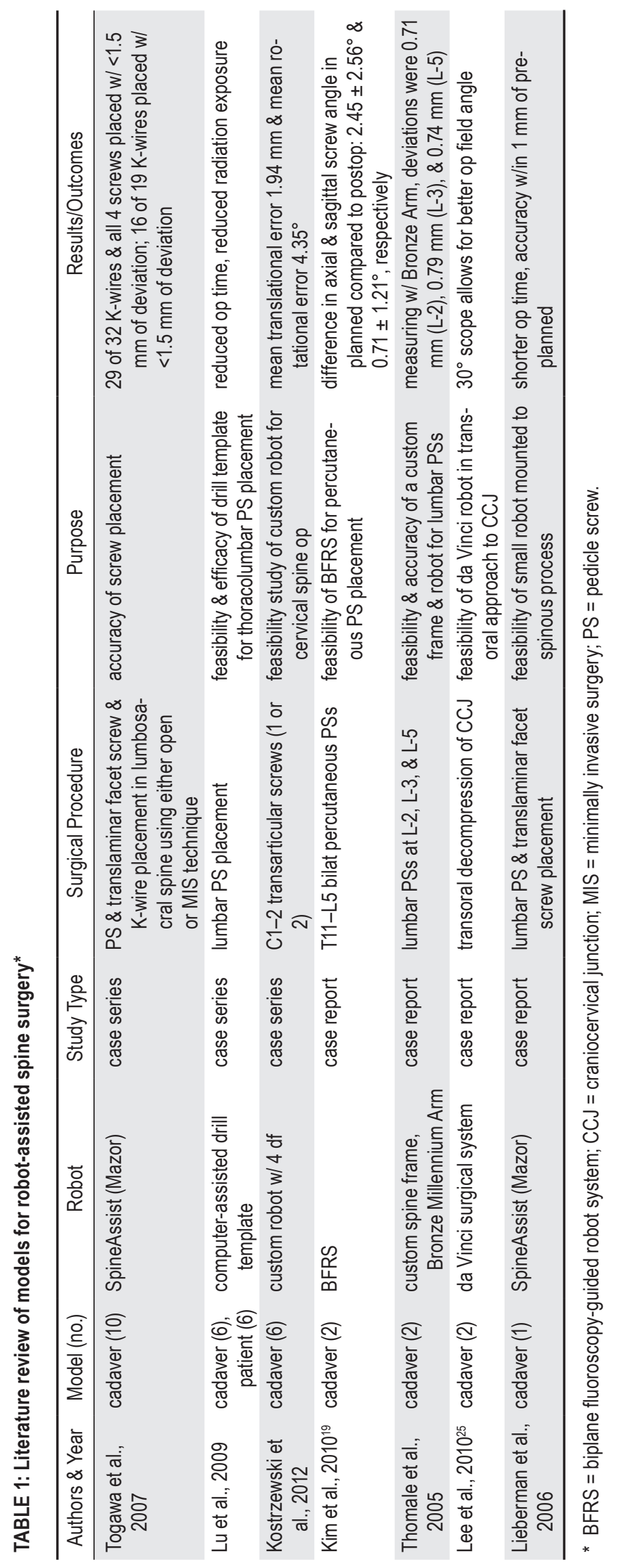


K-wires for which the SpineAssist Mazor robot was used in 10 cadavers. In that study a preoperative CT scan was obtained in each cadaveric torso, from which the 3D reconstructions were used for synchronization with the robot and surgical entry site planning. Multiple types of surgeries were performed, including K-wire placement with either an open or minimally invasive technique, pedicle screws with the PathFinder minimally invasive system, and translaminar placement of screws or K-wires completed using a Hover-T minimally invasive frame. Postoperatively, CT scans were performed to analyze accuracy of K-wire and screw placement. The results were divided based on their level of accuracy in regard to lateral, anteroposterior, and axial deviations on postoperative imaging compared with preoperative planning. High accuracy was defined as deviation within $1.5 \mathrm{~mm}$ of any axis, and low accuracy was a deviation $>1.5 \mathrm{~mm}$. The K-wires had an overall average deviation of $0.87 \pm 0.63 \mathrm{~mm}$ from preoperative planning, with no difference in open versus minimally invasive techniques. Pedicle screw placement was assessed separately; they had all been placed successfully, with an overall average deviation of $1.03 \pm 0.59$ $\mathrm{mm}$. In the translaminar facet instrumentation surgery, the overall average K-wire deviation was $1.05 \pm 0.56 \mathrm{~mm}$. This study showed good feasibility and accuracy for the use of the SpineAssist robot for placement of pedicle and translaminar instrumentation in the lumbosacral spine.

The 2 next largest studies used 6 cadavers and tested the feasibility of a novel spinal stereotaxy system with reverse-engineered drill templates based on CT scan $3 \mathrm{D}$ reconstruction of thoracolumbar vertebrae and $\mathrm{C} 1-2$ transarticular screw placement. ${ }^{21,27}$ In the thoracolumbar model, the drill templates had the advantage of being inexpensive (US \$20), requiring no extra equipment, and allowing for pedicle screw placement in 1-2 minutes. These findings were subsequently applied in 6 patients, with similar results. ${ }^{27}$ In the paper by Kostrzewski et al., ${ }^{21}$ the authors reported how they designed a robot that uses thin-slice CT scans with navigation software to automatically position the robot properly for the surgeon to place the C1-2 transarticular screws. Their study showed a total time from registration to screw placement of $17 \mathrm{~min}-$ utes, with translational screw errors averaging $1.94 \mathrm{~mm}$ (range 0.41-6.19 mm). These variables were equivalent to the surgeon's conventional nonrobot-assisted methods. ${ }^{21}$

Among the rest of the cadaveric studies in Table 1, none consisted of more than 2 cadavers. The procedures mostly investigated the feasibility of different spinal procedures by using a variety of robots. The different systems included the da Vinci surgical system as well as custom robot systems combined with navigation or fluoroscopy. Other procedures performed were mostly posterior lumbar pedicle screw instrumentation and 1 case report of a transoral decompression of the craniocervical junction. ${ }^{19,25,26,40}$

\section{Modern-Day Robotics and Studies With Spine Surgery}

Studies and Series Analyzing the SpineAssist System. Early case series of patients undergoing robotic spine surgery demonstrated the technical and surgical obstacles that needed to be addressed and overcome for successful wide-scale implementation in the operating room. ${ }^{2,39}$ In 2006, Barzilay et al. ${ }^{2}$ conducted a prospective trial of SpineAssist for lumbar fusion in 15 patients at 2 centers, in which 9 cases demonstrated either technical or clinical errors. Technical errors were attributed to improper registration of intraoperative fluoroscopic images with the preoperative CT scan. Clinical errors included excessive pressure on the guiding arm, which altered the trajectory of screw placement; inability of the robot to reach desired location; or improper attachment of the clamp to the spinous process. Sukovich et al. ${ }^{39}$ showed either full or partial success in 13 of 14 cases, with errors similarly attributed to increased soft-tissue pressure on the guiding arm and failure of registration. Additionally, software crashes and lengthy calculation time (9 seconds per screw) were noted as setbacks to be addressed in future development of the robotic technology, and the early version used in these studies has since been improved. 2,39

Since the implementation of robotic technology to the world of spine surgery, several case series and retrospective cohort studies on robot-assisted pedicle screw placement have demonstrated superior accuracy of screw placement when compared with prior estimates of accuracy in freehand and image-guided techniques. ${ }^{9,15}$ In a retrospective multicenter analysis of 842 patients by Devito et al., 3204 (98\%) of 3271 screws and guidewires were correctly placed with breaches $<2 \mathrm{~mm}$ according to fluoroscopy or the surgeon's clinical judgment. The Gertzbein and Robbins ${ }^{10}$ criterion for accuracy was used, which classifies a screw as 1) perfectly within the pedicle; 2) breaching $<2 \mathrm{~mm}$; 3) breaching between 2 and $4 \mathrm{~mm}$; or 4) breaching between 4 and $6 \mathrm{~mm}$. A postoperative CT scan was available for assessment of screw placement in 139 patients, and $98.3 \%$ of these screws were placed within $2 \mathrm{~mm}(89.3 \%$ completely within the pedicle and $9 \%$ breaching $<2 \mathrm{~mm}) .{ }^{9}$ Almost half $(49 \%)$ of the operations were performed percutaneously, and permanent nerve damage occurred in none of the patients.

The study by Devito et al. ${ }^{9}$ compared these error rates to a prior meta-analysis of conventional pedicle screw placement done by Kosmopoulos and Schizas ${ }^{20}$ in 2007, citing a $81.9 \%$ accuracy rate, although the analysis lacked a standard of accuracy for screw placement.9,20 The lack of postoperative CT scans for evaluation in the majority of patients studied is cause for criticism of the study., ${ }^{9,37}$ However, the increased rate of percutaneous procedures may reduce soft-tissue pressure and trajectory deviation, which may have led to superior results.9,12 Of note, many additional studies (Table 2) compared robotic screw malposition rates to the results of a study analyzing conventional screw placement by Hicks et al. in 2010. ${ }^{12}$ The systematic review of pedicle screw placement in pediatric patients found that $4.2 \%$ of screws were malpositioned, but when postoperative CT scans were measured for accuracy, $15.7 \%$ of screws were malpositioned. Although measurement techniques are not standardized, the estimated malposition rates of conventional screw placement in studies such as those conducted by Kosmopoulos and Schizas and Hicks et al. are much higher than those of current retrospective robotics studies. ${ }^{9,12,20}$ 
Robotics and the spine

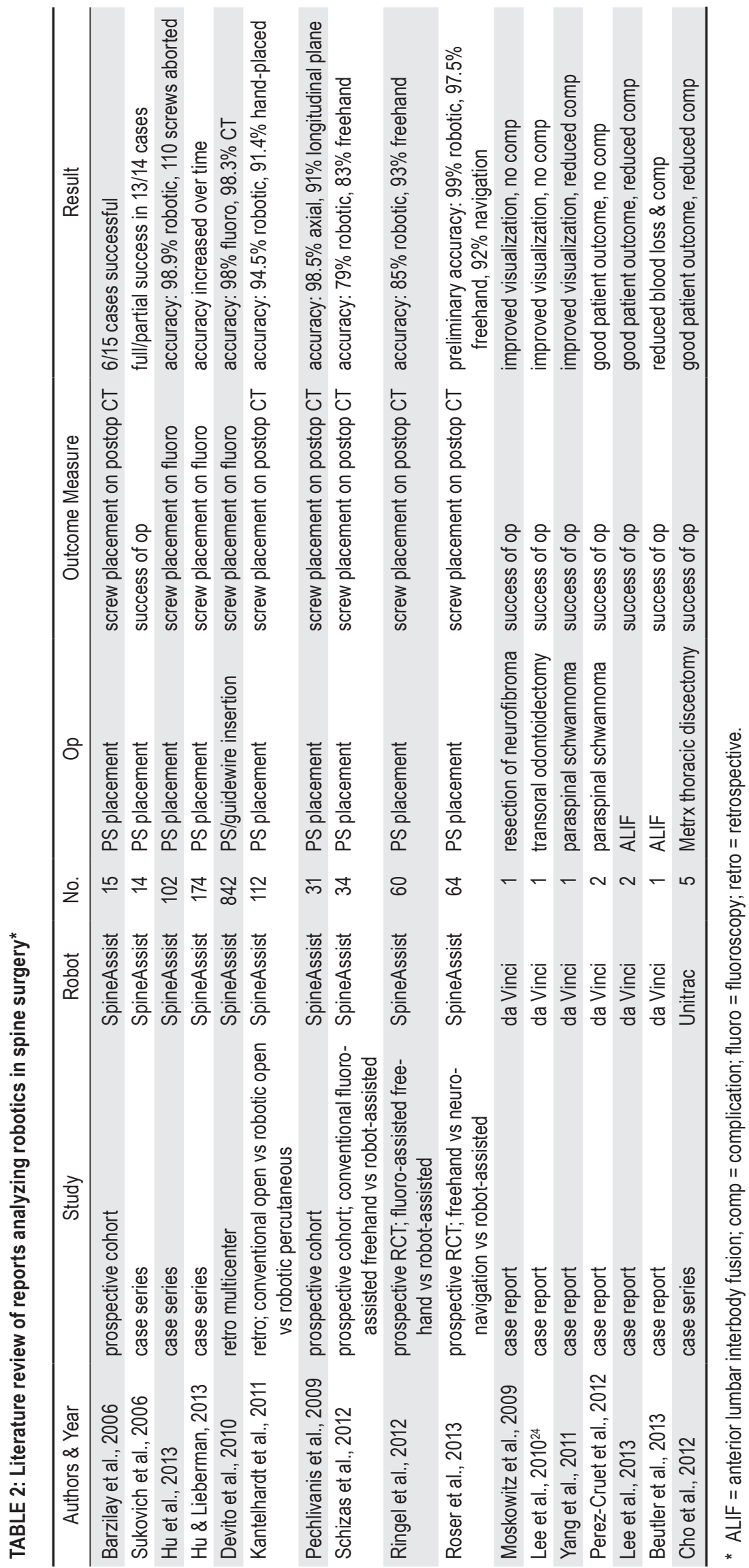




\section{F. Shweikeh et al.}

Hu et al. ${ }^{14}$ conducted another important case series of 102 consecutive patients in whom the SpineAssist was used, which yielded similarly high accuracy rates. In their investigation 949 (98.9\%) of 960 robotically placed screws were successfully positioned and 11 were malpositioned, presumably due to incorrect onset of trajectory. Robotic guidance was aborted for 110 screws, which were generally attributed to failure of the robot to register preoperative plans with intraoperative imaging, and/or technical problems with the planned trajectory. The majority of these aborted screws were in the thoracic $(61.8 \%)$ and lumbar spine (30\%), and each was converted to manual placement. The study was limited, however; the evaluation was done with fluoroscopy, and accuracy was characterized as either successfully placed or malpositioned. In 2011, Kantelhardt et al. ${ }^{15}$ compared the conventional open procedure to robotic open and robotic percutaneous techniques in a retrospective cohort analysis, and found that $94.5 \%$ of robot-assisted and $91.4 \%$ of conventionally placed screws were placed completely within the bone on postoperative CT scans $(\mathrm{p}=0.00001)$, whereas there was no significant difference between the open robotic versus percutaneous robotic procedure. Retrospective analyses of robot-assisted cohorts have overall demonstrated very high accuracy rates in large numbers of screw placements., ${ }^{9,14,15}$ Furthermore, in $2013 \mathrm{Hu}$ and Lieberman ${ }^{13}$ contended that the successful placement of pedicle screws with robotic assistance follows a learning curve that increases with surgeons' experience. In a consecutive cohort study of 174 patients who underwent pedicle screw placement by the same surgeon, accuracy rates increased from $82 \%$ in the first 30 patients to $93 \%$, $91 \%$, and $95 \%$, respectively, in the following groups of 30 patients. Although the learning curve may be different for an inexperienced surgeon, the study demonstrated that accuracy ratings may further improve with time.

Prospective cohort studies (Table 2) of patients who have undergone robot-assisted pedicle screw placement have shown conflicting results, demonstrating the need for further prospective and randomized studies for clarification. ${ }^{33,37}$ Pechlivanis et al. ${ }^{33}$ evaluated the SpineAssist in 31 patients, showing that $98.5 \%$ of pedicle screws were placed within $2 \mathrm{~mm}$ in the axial plane on postoperative CT scans, and $91 \%$ of screws were placed in the longitudinal plane. Schizas et al. ${ }^{37}$ studied 34 consecutive patients, comparing 64 screws placed with SpineAssist and 64 placed with conventional fluoroscopic guidance using the freehand technique. On postoperative CT scans, 79\% of robot-assisted screws (compared with $83 \%$ of fluoroscopy-guided freehand screws) were placed perfectly within pedicle margins. Because accuracy rates vary greatly between studies, it is important to consider variation in surgeon experience with the robot, accessibility in the operating room, and patient factors such as bone density, which may alter effectiveness data of early trials..$^{33,36}$

Currently, there are few prospective trials or randomized controlled trials (RCTs) comparing accuracy rates (Table 2). ${ }^{35-37}$ Ringel et al..$^{35}$ conducted a single-center prospective RCT for placement of lumbar and sacral pedicle screws comparing SpineAssist robot-assisted technique to freehand with fluoroscopic guidance in 60 patients. Accu- racy assessed using the Gertzbein and Robbins ${ }^{10}$ criterion on postoperative CT scans showed that $93 \%$ of freehand screws were correctly placed within $2 \mathrm{~mm}$ of the pedicle, compared with $85 \%$ in the robot-assisted group. This result is surprising, given that observational cohort studies have noted greatly increased accuracy., ${ }^{9,14,33}$ Although randomization and postoperative evaluation were accounted for, a criticism of the study points out that a bed mount was used for each patient, which may have led to increased relative motion between the patient and the bed, and therefore decreased accuracy. ${ }^{13,35}$ An additional randomized controlled prospective study done by Roser et al. ${ }^{36}$ compared conventional freehand versus standard open neuronavigation versus robot-assisted pedicle screw placement. Preliminary results in 37 patients have demonstrated $97.5 \%$ accuracy (completely within the pedicle) for freehand, 92\% accuracy for surface-matching neuronavigation, and 99\% accuracy for robot-assisted screws. The current data from randomized prospective trials are inconclusive and scarce, requiring further study and multicenter analysis from various experienced surgeons for further elucidation of the accuracy of pedicle screw placement. ${ }^{35-37}$ Nevertheless, Schizas et al. ${ }^{37}$ argued the importance of the fact that robots can place these screws as well or almost as well as an experienced surgeon, which may be important when combined with the potential advantages of decreased fatigue and radiation exposure. Improvements in implementation of these devices in spine surgery and more experience with the technique, when considered in light of the benefits of visualization and potential reduced radiation exposure afforded by this technology, will most likely provide better outcomes in spine surgery. 6,9

The da Vinci Surgical System: Spinal Applications. Although accuracy estimates are still debated, several case reports cite many advantages observed when expanding use of robotic assistance into spine operations. Established for general surgery in 2000, the da Vinci surgical system has been used with great success within the past few years in various spine procedures such as anterior lumbar interbody fusion, ${ }^{6,24}$ transoral odontoidectomy, ${ }^{30}$ resection of thoracolumbar neurofibroma, ${ }^{30}$ and resection of paraspinal schwannoma. ${ }^{34,35}$ Although case reports differ greatly in procedure and technique, there were several observations made in every case that proved beneficial for patient outcomes (Table 2). ${ }^{6,19,23,24,34,45} \mathrm{Vi}$ sualization was unanimously considered superior when using the robotic system. ${ }^{6,30,45}$ The increased magnification offered by robotic assistance provided improved visualization for careful dissection of associated nerves and blood vessels. ${ }^{3,12}$ The $3 \mathrm{D}$, high-definition capability was useful for visualization at the level of the anatomy. ${ }^{6,30}$ Beutler et al. ${ }^{6}$ specifically described the reduced morbidity created by a transperitoneal approach and maintenance of nearby structures allowed with the protection of the robotic system. Improved visualization and dissection of intraabdominal structures was directly related to benefits in patient outcomes. ${ }^{45}$ Lee et al. ${ }^{23}$ noted that this technique may lead to reduction in postsurgical complications such as urological or sexual dysfunction. Smaller incisions and reduced blood loss, lower morbidity and complications, 
reduced postoperative opioid use, shorter hospital stay, and rapid recovery were observed in multiple case studies. ${ }^{6,15,28,34}$

\section{Spine Biomechanics Testing}

In recent years, a number of robotic systems innovations have enabled in vitro biomechanical studies to approach conditions more closely approximating realworld, in vivo scenarios. Several of these innovations are summarized in Table 3. The literature describes 3 protocols for robotic control system design in biomechanical testing of the spine: stiffness, or position-controlled; flexibility, or load-controlled; and hybrid-controlled testing. ${ }^{31,32}$ Early robotic controllers consisted of positioncontrolled devices that simply made motor-controlled displacement along 3 or $6 \mathrm{df}$. The disadvantage of such simple control systems is that the actual loads can be prescribed only indirectly to a specimen by calculating from the positional displacements. A key goal of recent robotic systems development, therefore, has been to improve on the present state of the art to achieve continuous, realtime, load-controlled or hybrid-controlled (as opposed to position-controlled) testing and to apply it in various scenarios approximating physiological ranges of motion (ROMs). Another important advantage of robot-based spinal testing systems is multiaxial motion, which allows for reorienting force vectors in real time to better simulate in vivo conditions.

The selected innovations highlighted in Table 3 show considerable progress in the design of robotic systems for biomechanical spine testing over the past decade. Tian $^{41}$ introduced fuzzy logic control in robotic software as an improved alternative to hybrid control. Walker and Dickey ${ }^{43}$ provided an early demonstration of a parallel robotic system in which $6 \mathrm{df}$ was used for biomechanical joint testing, which improves on serial robot designs by enabling complex 3D loading patterns and facilitating studies on specimens with poorly understood loaddeformation properties. Schulze et al. ${ }^{38}$ demonstrated the ingenious combination of a multisegment robotic spine testing system with optical markers that can be affixed to rigid points on the specimen. With the aid of a camera, the relative motion of these optical markers can be used to ascertain the load-deformation characteristics of a complex specimen, such as a spinal segment with multiple joints. Two papers from the Memphis group ${ }^{4,17}$ highlight the development and application of a novel cartesian-based real-time load-controlled robotic testing system. Their validating experimental application exemplifies the advantages of robotic testing over nonrobotic platforms; namely to apply a dynamically oriented force. Their system is capable of applying a real-time dynamically reorienting force that follows the midline of the intervertebral disc as the joint of the specimen moves with rotation and deformation, which allows rigorous testing throughout the physiological ROM. Bell et al. ${ }^{3}$ developed a realistic, adaptive control algorithm based on a hybridcontrolled model that was found to minimize off-axis forces and increase the size of the neutral zone and ROM. Last, Martínez et al. ${ }^{29}$ demonstrated a direct force control method that improves on position-controlled and load- controlled methods and is ideally suited for multisegment testing.

Robotic spinal biomechanical testing systems, like their surgical counterparts, remain in the early phases of innovation. At this time, it is unclear which integrated testing system will become the gold standard and which innovations will prove essential to future work. Moreover, the field of spine surgery still awaits the first dramatic clinical innovation to derive directly from the use of improved robotic biomechanical testing platforms. In the next decade, as robots enable biomechanical testing systems to model in vivo reality more closely, there is the promise of more rapid and effective testing of devices and procedures that will improve patient care. Moreover, innovations in robotic biomechanical testing systems may provide much-needed synergy for the tandem evolution of intraoperative robots for spine surgery.

\section{Discussion}

From the standpoint of future studies in robot-assisted spine surgery, the focus should be to explore those issues that limit the technology from widespread adoption. Certainly the major categories that encompass the limitations in robotic adoption revolve around cost, lack of advanced tools, and time efficiency. These factors in many ways are interrelated and can conceivably be studied in a single encompassing prospective or retrospective study. Advancements in each category on a "micro" level can lead to improvements on a "macro" analysis. For instance, the technology that needs to be explored on a "micro" level would be development of discectomy tools, drill attachments, and modularity of the robotic arm that can interchange various specialized tools in a quick and efficient manner. Once these tools are developed, the robotic process could far exceed the precision and accuracy of the human hand. Clinical studies on tumor resection and difficult spinal access areas (transforaminal or posterior lumbar interbody fusion, costotransvertebral corpectomy, pedicle subtraction osteotomies, and so on) could potentially highlight the superiority of robotic assistance.

In addition to the advantage of visualization, the freedom of motion provided by the robotic arms was comparable to that of human hands, and steadiness was considered superior to human hand performance. ${ }^{8,30,45}$ Cho et al. ${ }^{8}$ used a robotic arm (Unitrac; Aesculap) during an oblique paraspinal approach for thoracic disc herniation with a tubular retractor. ${ }^{27}$ Their case series of 5 patients demonstrated the advantages of using the robotic arm as a pneumatic holding device; the surgery was more feasible with the robot's stronger holding power and stiffer angle. Yang et $\mathrm{al}^{45}$ considered the robot-assisted retroperitoneal approach to resection of paraspinal schwannoma to be superior to the conventional technique in minimally invasive access as well as safer for the patient. Superior control in a narrow working space at great depth was noted for transoral odontoidectomy. ${ }^{24}$ As these case studies demonstrate, there is potential for application of this technology to such high-risk, minimally invasive operations.

At the same time, many of the listed studies have noted obstacles with robotic assistance that the investigators 
F. Shweikeh et al.

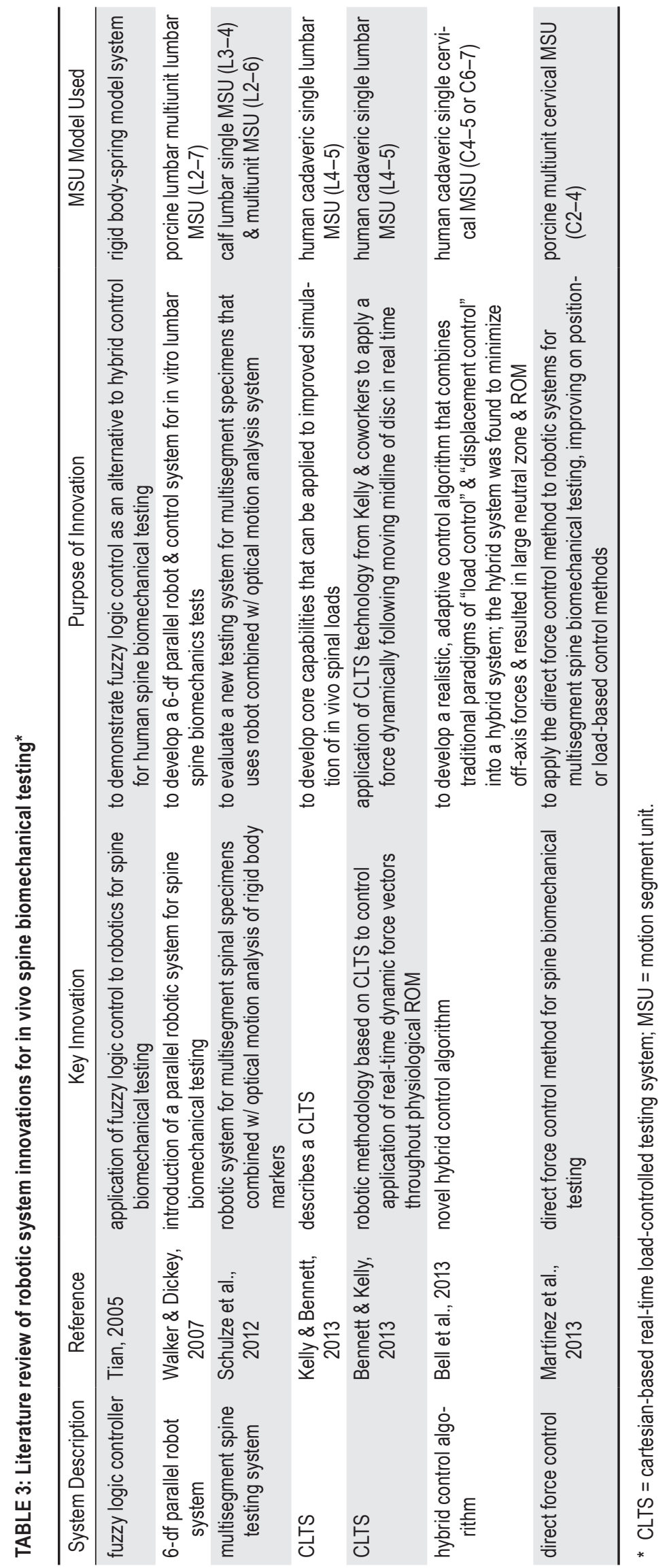


thought should be overcome before its implementation into mainstream spine surgery. ${ }^{6,23,24}$ The cost of robotic technology was cited as one of the major obstacles to its feasibility and consistent use across multiple institutions. ${ }^{5,23}$ Other noted difficulties have been logistical in nature; it is technically challenging and time-consuming to set up an operating room while coordinating a robot, imaging, and working without a team trained and capable of handling its use..$^{6,23}$ Also significant, the robot lacks the tools for bony dissection or discectomy, and therefore these portions of the case had to be performed around the robot. ${ }^{23,24}$ Training a surgical team to use and work around the robot requires a considerable amount of time and expense, which would require significant patient benefits to outweigh these setbacks..$^{6,24}$

A ubiquitous observation throughout robot-assisted surgery studies is the increased time required for both preoperative planning and intraoperative use. ${ }^{35,36,45}$ Ringel et al. ${ }^{35}$ noted a significant increase both in planning time for screw trajectories (24 minutes) and in surgical time (11 minutes). Additional studies cited increased preand intraoperative time relative to freehand techniques in screw placement and tumor resection. ${ }^{36,45}$ The general consensus, however, was that this increased time may be reduced by a learning curve for each surgeon using a new device. $^{13,45}$

Reduction in radiation exposure is one of the major theoretical advantages that robotics may provide to minimally invasive spine surgery. ${ }^{2,5}$ According to the prospective RCT conducted by Ringel et al., ${ }^{35}$ there was no significant difference in radiation exposure between robot-assisted and freehand techniques. When including preoperative planning CT scanning for the robotic procedure (which may differ by level and protocol), this study claimed that a higher dose of radiation was incurred by the patient. Similarly, Schizas et al. ${ }^{37}$ demonstrated no significant difference in radiation between the 2 groups in a prospective cohort study. In contrast, Kantelhardt et al. ${ }^{15}$ retrospectively observed that the average x-ray exposure per screw was 77 seconds for conventional procedures, and 34 seconds in robot-guided procedures. Roser et al. ${ }^{36}$ noted a similar decrease in radiation by almost half in the robot-assisted group compared with the freehand group in a prospective RCT. Because both retrospective and prospective studies show contradictory results, this is another aspect of robot-assisted spine surgery that needs further investigation to resolve the discrepancies.

An emphasis on workflow and design must also be at the forefront of future robot-assisted spine surgery. As great as the focus is on engineering and technology development in robots, there must be an equal emphasis on design and logistics. The success of the technology is dependent on the ease of use and increased efficiency that it can offer the surgeon. Studies looking at low-profile, flexible robotic arms, or even robotic arms that are attached to the ceiling as opposed to the operating table or a cumbersome base frame are examples of designs that can be further explored. The incorporation of image-guided navigation and electrophysiological neuromonitoring into robotic arms to "de-clutter" the operative field is another example of how design studies can improve the surgeon's experience. From a workflow standpoint, so-called simultaneous robotic spine surgery, in which a preprogrammed robotic arm instruments the bony pedicle spine while the surgeon is simultaneously executing a laminar decompression, presents a novel concept that appears very achievable with current robotic arm technology. Ultimately, these types of future studies could translate into decreased operating times, less blood loss, faster recoveries, and ultimately better clinical outcomes to justify the overall costs.

\section{Conclusions}

There are numerous potential advantages and limitations to robotic spine surgery, as has been suggested in retrospective and prospective studies, and in case reports in the literature. In terms of accuracy, retrospective analyses have indicated accuracy rates comparable to those with conventional techniques, which may even be significantly lower. Randomized trials are few in number and show conflicting results regarding accuracy; these results require further investigation to resolve the discrepancies. The various advantages of robotics are improved intraoperative visualization and dissection, smaller incisions, reduced blood loss, reduced recovery time, and shorter hospital stay. The disadvantages that have been noted, although perhaps surmountable with future technological improvements, include the need for greater surgeon experience, cost, training time, and operating room dynamics. Given the potential advantages of robotic technology for improvements in spine surgery, spine biomechanics testing, and patient outcomes, more research is needed to study the applicability of this technology in the spinal operating room. Future research should include large multicenter RCTs with multiple surgeons and a standardized accuracy rating system, such as postoperative CT imaging. Due to the substantial costs of the robotic equipment and the additional associated training necessary, research is needed to substantiate that the increased costs will result in significant benefits that will justify the considerable expense.

\section{Disclosure}

Dr. Kim is a consultant for DePuy Synthes. The authors report no conflict of interest concerning the materials or methods used in this study or the findings specified in this paper.

Author contributions to the study and manuscript preparation include the following. Conception and design: Drazin. Analysis and interpretation of data: Drazin, Shweikeh, Arnell, Barnard. Drafting the article: Drazin, Shweikeh, Amadio, Arnell, Barnard. Critically revising the article: Drazin, Shweikeh, Kim, Johnson. Reviewed submitted version of manuscript: Drazin, Arnell, Kim, Johnson. Approved the final version of the manuscript on behalf of all authors: Drazin. Study supervision: Johnson.

\section{References}

1. Ahmed K, Ibrahim A, Wang TT, Khan N, Challacombe B, Khan MS, et al: Assessing the cost effectiveness of robotics in urological surgery-a systematic review. BJU Int 110:15441556, 2012

2. Barzilay Y, Liebergall M, Fridlander A, Knoller N: Miniature 
robotic guidance for spine surgery-introduction of a novel system and analysis of challenges encountered during the clinical development phase at two spine centres. Int J Med Robot 2: 146-153, 2006

3. Bell KM, Hartman RA, Gilbertson LG, Kang JD: In vitro spine testing using a robot-based testing system: comparison of displacement control and "hybrid control." J Biomech 46: 1663-1669, 2013

4. Bennett CR, Kelly BP: Robotic application of a dynamic resultant force vector using real-time load-control: simulation of an ideal follower load on cadaveric L4-L5 segments. J Biomech 46:2087-2092, 2013

5. Bertelsen A, Melo J, Sánchez E, Borro D: A review of surgical robots for spinal interventions. Int J Med Robot 9:407-422, 2013

6. Beutler WJ, Peppelman WC, Dimarco LA: The da Vinci robotic surgical assisted anterior lumbar interbody fusion: technical development and case report. Spine (Phila Pa 1976) 38:356363,2013

7. Cahill KS, Wang MY: Evaluating the accuracy of robotic assistance in spine surgery. Neurosurgery 71:N20-N21, 2012

8. Cho JY, Lee SH, Jang SH, Lee HY: Oblique paraspinal approach for thoracic disc herniations using tubular retractor with robotic holder: a technical note. Eur Spine J 21:2620 2625,2012

9. Devito DP, Kaplan L, Dietl R, Pfeiffer M, Horne D, Silberstein B, et al: Clinical acceptance and accuracy assessment of spinal implants guided with SpineAssist surgical robot: retrospective study. Spine (Phila Pa 1976) 35:2109-2115, 2010

10. Gertzbein SD, Robbins SE: Accuracy of pedicular screw placement in vivo. Spine (Phila Pa 1976) 15:11-14, 1990

11. Hamilton DK, Smith JS, Sansur CA, Glassman SD, Ames CP, Berven SH, et al: Rates of new neurological deficit associated with spine surgery based on 108,419 procedures: a report of the scoliosis research society morbidity and mortality committee. Spine (Phila Pa 1976) 36:1218-1228, 2011

12. Hicks JM, Singla A, Shen FH, Arlet V: Complications of pedicle screw fixation in scoliosis surgery: a systematic review. Spine (Phila Pa 1976) 35:E465-E470, 2010

13. Hu X, Lieberman IH: What is the learning curve for roboticassisted pedicle screw placement in spine surgery? Clin Orthop Relat Res [epub ahead of print], 2013

14. Hu X, Ohnmeiss DD, Lieberman IH: Robotic-assisted pedicle screw placement: lessons learned from the first 102 patients. Eur Spine J 22:661-666, 2013

15. Kantelhardt SR, Martinez R, Baerwinkel S, Burger R, Giese A, Rohde V: Perioperative course and accuracy of screw positioning in conventional, open robotic-guided and percutaneous robotic-guided, pedicle screw placement. Eur Spine J 20:860-868, 2011

16. Kazemi N, Crew LK, Tredway TL: The future of spine surgery: new horizons in the treatment of spinal disorders. Surg Neurol Int 4 (Suppl 1):S15-S21, 2013

17. Kelly BP, Bennett CR: Design and validation of a novel Cartesian biomechanical testing system with coordinated 6DOF real-time load control: application to the lumbar spine (L1-S, L4-L5). J Biomech 46:1948-1954, 2013

18. Kim MJ, Ha Y, Yang MS, Do HY, Kim KN, Kim H, et al: Robot-assisted anterior lumbar interbody fusion (ALIF) using retroperitoneal approach. Acta Neurochir (Wien) 152:675-679, 2010

19. Kim S, Chung J, Yi BJ, Kim YS: An assistive image-guided surgical robot system using $\mathrm{O}$-arm fluoroscopy for pedicle screw insertion: preliminary and cadaveric study. Neurosurgery 67:1757-1767, 2010

20. Kosmopoulos V, Schizas C: Pedicle screw placement accuracy: a meta-analysis. Spine (Phila Pa 1976) 32:E111-E1120, 2007

21. Kostrzewski S, Duff JM, Baur C, Olszewski M: Robotic sys- tem for cervical spine surgery. Int J Med Robot 8:184-190, 2012

22. Lau S, Vaknin Z, Ramana-Kumar AV, Halliday D, Franco EL, Gotlieb WH: Outcomes and cost comparisons after introducing a robotics program for endometrial cancer surgery. Obstet Gynecol 119:717-724, 2012

23. Lee JY, Bhowmick DA, Eun DD, Welch WC: Minimally invasive, robot-assisted, anterior lumbar interbody fusion: a technical note. J Neurol Surg A Cent Eur Neurosurg 74:258261, 2013

24. Lee JY, Lega B, Bhowmick D, Newman JG, O’Malley BW Jr, Weinstein GS, et al: Da Vinci robot-assisted transoral odontoidectomy for basilar invagination. ORL J Otorhinolaryngol Relat Spec 72:91-95, 2010

25. Lee JY, O’Malley BW, Newman JG, Weinstein GS, Lega B, Diaz J, et al: Transoral robotic surgery of craniocervical junction and atlantoaxial spine: a cadaveric study. Laboratory investigation. J Neurosurg Spine 12:13-18, 2010

26. Lieberman IH, Togawa D, Kayanja MM, Reinhardt MK, Friedlander A, Knoller N, et al: Bone-mounted miniature robotic guidance for pedicle screw and translaminar facet screw placement: Part I-Technical development and a test case result. Neurosurgery 59:641-650, 2006

27. Lu S, Xu YQ, Zhang YZ, Li YB, Xie L, Shi JH, et al: A novel computer-assisted drill guide template for lumbar pedicle screw placement: a cadaveric and clinical study. Int J Med Robot 5:184-191, 2009

28. Marano A, Choi YY, Hyung WJ, Kim YM, Kim J, Noh SH: Robotic versus laparoscopic versus open gastrectomy: a metaanalysis. J Gastric Cancer 13:136-148, 2013

29. Martínez H, Obst T, Ulbrich H, Burgkart R: A novel application of direct force control to perform in-vitro biomechanical tests using robotic technology. J Biomech 46:1379-1382, 2013

30. Moskowitz RM, Young JL, Box GN, Paré LS, Clayman RV: Retroperitoneal transdiaphragmatic robotic-assisted laparoscopic resection of a left thoracolumbar neurofibroma. JSLS 13:64-68, 2009

31. Panjabi MM: Biomechanical evaluation of spinal fixation devices: I. A conceptual framework. Spine (Phila Pa 1976) 13: 1129-1134, 1988

32. Panjabi MM: Hybrid multidirectional test method to evaluate spinal adjacent-level effects. Clin Biomech (Bristol, Avon) 22:257-265, 2007

33. Pechlivanis I, Kiriyanthan G, Engelhardt M, Scholz M, Lücke S, Harders A, et al: Percutaneous placement of pedicle screws in the lumbar spine using a bone mounted miniature robotic system: first experiences and accuracy of screw placement. Spine (Phila Pa 1976) 34:392-398, 2009

34. Perez-Cruet MJ, Welsh RJ, Hussain NS, Begun EM, Lin J, Park P: Use of the da Vinci minimally invasive robotic system for resection of a complicated paraspinal schwannoma with thoracic extension: case report. Neurosurgery 71 (1 Suppl Operative):209-214, 2012

35. Ringel F, Stüer C, Reinke A, Preuss A, Behr M, Auer F, et al: Accuracy of robot-assisted placement of lumbar and sacral pedicle screws: a prospective randomized comparison to conventional freehand screw implantation. Spine (Phila Pa 1976) 37:E496-E501, 2012

36. Roser F, Tatagiba M, Maier G: Spinal robotics: current applications and future perspectives. Neurosurgery 72 (Suppl 1): $12-18,2013$

37. Schizas C, Thein E, Kwiatkowski B, Kulik G: Pedicle screw insertion: robotic assistance versus conventional C-arm fluoroscopy. Acta Orthop Belg 78:240-245, 2012

38. Schulze M, Hartensuer R, Gehweiler D, Hölscher U, Raschke MJ, Vordemvenne T: Evaluation of a robot-assisted testing system for multisegmental spine specimens. J Biomech 45: 1457-1462, 2012 


\section{Robotics and the spine}

39. Sukovich W, Brink-Danan S, Hardenbrook M: Miniature robotic guidance for pedicle screw placement in posterior spinal fusion: early clinical experience with the SpineAssist. Int J Med Robot 2:114-122, 2006

40. Thomale UW, Kneissler M, Hein A, Maetzig M, Kroppenstedt SN, Lueth T, et al: A spine frame for intra-operative fixation to increase accuracy in spinal navigation and robotics. Comput Aided Surg 10:151-155, 2005

41. Tian L: An intelligent control method based on fuzzy logic for a robotic testing system for the human spine. J Biomech Eng 127:807-812, 2005

42. Togawa D, Kayanja MM, Reinhardt MK, Shoham M, Balter A, Friedlander A, et al: Bone-mounted miniature robotic guidance for pedicle screw and translaminar facet screw placement: part 2-evaluation of system accuracy. Neurosurgery 60 (2 Suppl 1):ONS129-ONS139, 2007

43. Walker MR, Dickey JP: New methodology for multi-dimen- sional spinal joint testing with a parallel robot. Med Biol Eng Comput 45:297-304, 2007

44. Wedmid A, Llukani E, Lee DI: Future perspectives in robotic surgery. BJU Int 108:1028-1036, 2011

45. Yang MS, Kim KN, Do HY, Pennant W, Ha Y: Robot-assisted resection of paraspinal Schwannoma. J Korean Med Sci 26: $150-153,2011$

Manuscript submitted November 19, 2013.

Accepted January 17, 2014.

Please include this information when citing this paper: DOI: 10.3171/2014.1.FOCUS13526.

Address correspondence to: Doniel Drazin, M.D., Department of Neurosurgery, Cedars-Sinai Medical Center, Maxine Dunitz Neurosurgical Institute, 8631 W. Third St., Ste. 800E, Los Angeles, CA 90048. email: doniel.drazin@cshs.org. 